\title{
Rare Cavitary MAC Lung Disease Causing a Serious Broncho-Pleural Air Leak in an Immune Competent Patient
}

\author{
Abdelkarim Waness
}

\begin{abstract}
Mycobacterium avium-intracellulare complex (MAC) is a nontuberculous mycobacterium that can cause a variety of pathologies in both immune-competent and immune-deficient individuals. In this paper, I present a rare and unique case of severe MAC cavitary pulmonary disease complicated by a serious broncho-pleural air leak and persistent hydropneumothorax in a patient with normal immunity. His successful recovery took a lengthy antibiotic course and a segmental lung resection with pleural decortication. A brief discussion about MAC lung disease follows this presentation.
\end{abstract}

Keywords: Mycobacterium avium-intracellulare complex; Cavitary lung disease; Broncho-pleural fistula; Hydropneumothorax; Segmentectomy; Pleural decortication

\section{Introduction}

Non-tuberculous mycobacteria, also known as atypical mycobacteria, are occasional human pathogens. These include: Mycobacterium avium-intracellulare complex (MAC), M. marinum, M. kansasii, and M. ulcerans. They usually infect individuals with decreased or blunted immunity but can be encountered in immunocompetent people. The spectrum of their pathologies includes lung infection, soft tissue disease, lymphatic involvement, and joint infection among others. In this paper, I am presenting a complicated case of MAC pulmonary disease in a person not known to have any immunodeficiency.

\section{Manuscript accepted for publication November 8, 2013}

Department of Medicine, Mafraq Hospital, PO Box 2951, Abu Dhabi, UAE.Email: awaness@mafraqhospital.ae

doi: http://dx.doi.org/10.4021/jmc1578w

\section{Case Report}

A 36-year-old diabetic man, on insulin replacement, was admitted with a 3-day history of left-sided chest pain and minimally productive cough. Clinically, the patient was mildly distressed. He had a temperature of $36.5^{\circ} \mathrm{C}$, respiration of $38 / \mathrm{min}$, pulse of $119 / \mathrm{min}$, BP of $128 / 79 \mathrm{mmHg}$, and $\mathrm{SPO}_{2}$ of $97 \%$ on room air. Auscultation showed left-sided reduced air entry and tachycardia. No palpable lymph nodes were found. The rest of the physical examination was unremarkable. Laboratory findings include: sodium $119 \mathrm{mmol} / \mathrm{L}$ (135 - $145 \mathrm{mmol} / \mathrm{L})$, potassium $4.5 \mathrm{mmol} / \mathrm{L}(3.4-5.1 \mathrm{mmol} / \mathrm{L})$, chloride $80 \mathrm{mmol} / \mathrm{L}(98-107 \mathrm{mmol} / \mathrm{L})$, WBC $16.6 \times 10^{9} / \mathrm{L}$ (range $\left.4.5-10 \times 10^{9} / \mathrm{L}\right)$, hemoglobin $16 \mathrm{~g} / \mathrm{L}(117-155 \mathrm{~g} / \mathrm{L})$, and platelets $631 \times 10^{9} / \mathrm{L}\left(140-400 \times 10^{9} / \mathrm{L}\right)$. HIV serology was negative. Initial chest X-ray (CXR) discovered a large left hydropneumothorax, a completely collapsed left lung containing a large cavity, and severe right mediastinal shift (Fig. 1). A chest tomography (CT chest) confirmed these

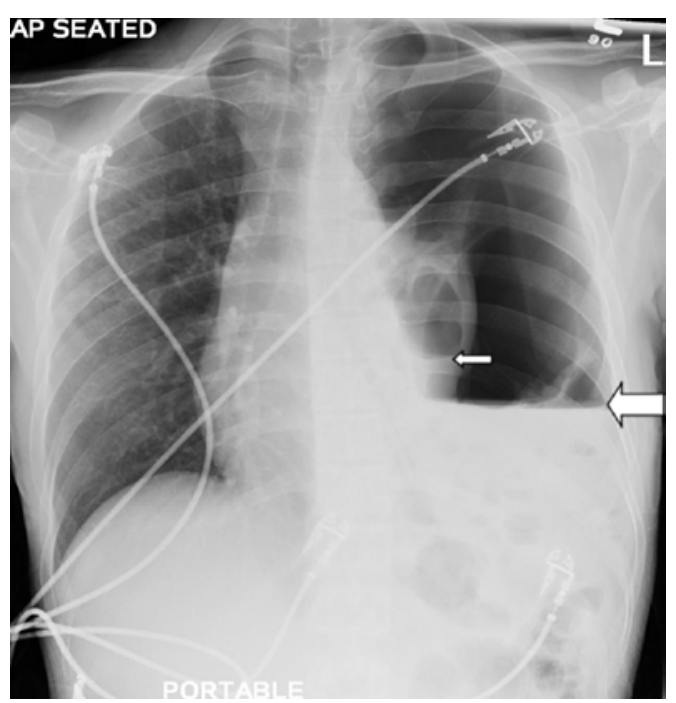

Figure 1. CXR showing large left hydropneumothorax with marked air-fluid level (large arrow), a collapsed left lung containing a cavity with another smaller air-fluid level (small arrow), and severe right mediastinal shift. 


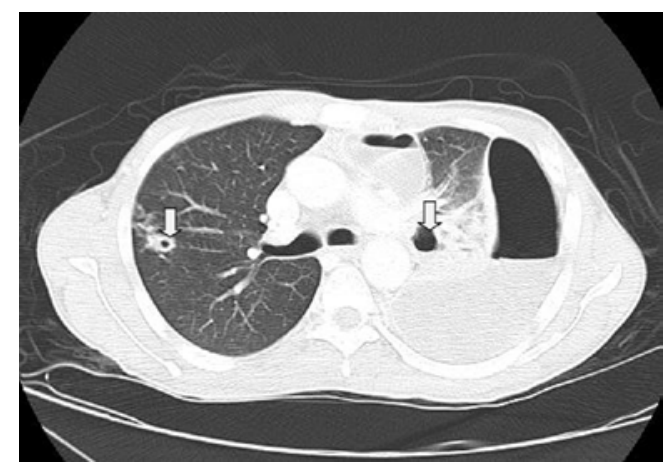

Figure 2. CT chest showing large left hydropneumothorax with air-fluid level and multiple bilateral pulmonary cavities (arrows).

findings and demonstrated other smaller cavitary lesions in both lungs (Fig. 2).

A large-bore chest tube was placed removing more than $500 \mathrm{~mL}$ of turbid pleural fluid that was negative for acid fast bacilli stains. Polymerase chain reaction (PCR) testing came back positive for MAC. A second PCR test confirmed the same microorganism.

The patient continued having dyspnea and tachycardia. Repeat CXRs showed persistent hydropneumothorax though smaller in size despite replacing chest drains. Subsequently bronchoscopy with broncho-alveolar lavage (BAL) was done. It confirmed the presence of a broncho-pleural fistula causing an expiratory air leak into the left pleural space. Fluid culture from BAL grew MAC. The patient was started on azithromycin, rifampin, and ethambutol. After protracted medical management for 2 months, he underwent successful left lung segmental resection and pleural decortication. $\mathrm{He}$ was discharged to finish his antibiotic course in the outpatient setting.

\section{Discussion}

MAC is a ubiquitous non-tuberculous mycobacterium acquired via inhalation or ingestion. It can affect both immunocompetent and immunocompromised individuals [1]. MAC can infect many body organs such as lungs, liver, and spleen. In the respiratory system, it can cause a variety of diseases including: pneumonia, bronchiectasis, lung abscess/cavity, empyema, and pneumothorax [2].

Clinicians must be aware of possible MAC lung disease and pulmonary tuberculosis co-existence. Some authors advocate repeating a positive MAC PCR before embarking on treatment [3]. Rarely, MAC can cause severe hydropneumothorax from a ruptured lung cavity or broncho-pleural fistula as in this case. A multidisciplinary approach is usually needed in the management of MAC lung disease. This pathogen remains reasonably sensitive to few antibiotics (macrolides, quinolones, and so on) and antituberculous drugs (rifampin, ethambutol, and so on). It is usually treated with a drug combination for a period of 12 months $[4,5]$. In case of MAC lung disease with broncho-pleural or alveolar-pleural fistulas, endobronchial valves insertion can be attempted by experienced pulmonologists [6]. In advanced non-tuberculous mycobacterial pleuro-pulmonary disease, surgical interventions such as segmentectomy, lobectomy, pneumonectomy, or pleural decortication might be required with varying degree of morbidity and mortality $[7,8]$.

\section{Conclusion}

MAC can cause severe pleuro-pulmonary disease in both immune-competent and immune-suppressed individuals. The diagnosis is confirmed by a combination of positive PCR test (preferably two tests), and cultures. Intractable hydropneumothorax is a dreaded complication that requires a lengthy multi-disciplinary intervention. MAC remains sensitive to quite a few drugs even though duration of therapy is lengthy. Finally, clinicians must pay special attention to possible MAC and Mycobacterium tuberculosis co-existence since therapeutic choices might change.

\section{Conflict of Interest}

None.

\section{Funding Source}

Nil.

\section{References}

1. Kasperbauer SH, Daley CL. Diagnosis and treatment of infections due to Mycobacterium avium complex. Semin Respir Crit Care Med. 2008;29(5):569-576.

2. Haider A, Schliep T, Zeana C. Nontuberculous mycobacterium disease with pleural empyema in a patient with advanced AIDS. Am J Med Sci. 2009;338(5):418420.

3. Takeda M, Ito W, Kobayashi N, Konno K, Takahashi T, Tatsuko R, Tomita N, et al. Co-existence of Mycobacterium tuberculosis and Mycobacterium intracellulare in one sputum sample. Intern Med. 2008;47(11):10571060.

4. Park SU, Koh WJ, Kwon OJ, Park HY, Jun HJ, Joo EJ, Lee NY, et al. Acute pneumonia and empyema caused by Mycobacterium intracellulare. Intern Med. 2006;45(17):1007-1010.

5. Koirala J. Mycobacterium Avium-Intracellulare Treat- 
ment \& Management. http://emedicine.medscape.com/ article/222664-treatment. Accessed January 10, 2012.

6. El-Sameed Y, Waness A, Al Shamsi I, Mehta AC. Endobronchial valves in the management of broncho-pleural and alveolo-pleural fistulae. Lung. 2012;190(3):347-351.

7. Mitchell JD, Bishop A, Cafaro A, Weyant MJ, Pomerantz $\mathrm{M}$. Anatomic lung resection for nontuberculous my- cobacterial disease. Ann Thorac Surg. 2008;85(6):18871892; discussion 1892-1883.

8. Kawamoto H, Yamagata M, Nakashima H, Kambe M, Okamoto N, Yamane K, Takahashi H, et al. Development of a case of Mycobacterium avium complex disease from right pleural effusion. Nihon Kokyuki Gakkai Zasshi. 2000;38(9):706-709. 